Neurosurg Focus 4 (6): Article 11, 1998

\title{
Evidence-based review of the role of reoperation in the management of malignant glioma
}

\section{Clifford B. Soults, M.D., Greg S. Canute, M.D., and Timothy C. Ryken, M.D.}

Department of Neurosurgery, University Hospital, State University of New York Health Science Center, Syracuse, New York; and Division of Neurosurgery, Department of Surgery, University of Iowa Hospitals and Clinics, Iowa City, Iowa

Using an evidence-based approach to available clinical studies, the authors examined the role of reoperation in the management of malignant glioma. A review of 1270 Medline-referenced articles spanning the period from 1966 through March 1998 was undertaken using the key words "glioblastoma" and "astrocytoma." Using an evidence-based four-tiered grading system, the authors found only 14 articles that met their inclusion criteria. Of these, 11 were graded as Class III (retrospective case series) and three as Class II (prospective nonrandomized studies). There were no Class I reports (randomized clinical trials), and all Class IV reports (opinion reports) were excluded. The authors of 10 Class III and one Class II articles supported the role of reoperation in increasing survival time or quality of life in selected patients; however, the results of multivariate analysis in two Class II and one Class III article did not support prolonged survival. The authors conclude that there was insufficient evidence to support either a standard or a guideline for reoperation in malignant glioma given the current status of the literature. Selection bias was a major factor in these studies. With continued interest in clinical trials for recurrent malignant glioma, the role of reoperation needs to be addressed in case-controlled or randomized fashion to establish either standards or guidelines on this commonly debated issue.

Key Words * glioma * reoperation * guidelines * practice parameters

Despite advances in molecular genetics and increasingly sophisticated treatment strategies, the survival data for patients with malignant glioma has changed little in the last 20 years. Patients generally undergo surgical resection coupled with radiation therapy and adjuvant chemotherapy. With rare exception, these lesions recur locally, and consideration of additional treatment is required. Depending on the patients' status and the opinion of the surgeon, patients often undergo a second cytoreductive procedure that may be coupled with another form of adjuvant therapy. Although this is a common clinical practice, there is relatively little scientific evidence documenting the effects of a second operation. To evaluate the role of a second operation in management of glioblastoma (GBM), we have examined the available literature on reoperation procedures using an evidence-based technique. 
Evidence-based guidelines have been used in a variety of clinical fields as a method of building the scientific foundation of medical practice in a particular area of study.[1,15] We attempted to address the question: Does reoperation for malignant glioma at the time of recurrence improve life expectancy and/or quality of life? Expecting relatively few studies addressing this issue, we defined our inclusion criteria rather broadly. We proposed to include all Class I, II, and III studies that provided survival and/or quality-of-life measures in at least 10 patients undergoing a second cytoreductive surgical procedure in whom a diagnosis of malignant glioma was made at the time of the second operation (World Health Organization Grades III and IV). A Medline literature search was made for articles written during the period from 1966 through March 1998, by using the key words "astrocytoma" or "glioblastoma," and cross referenced with the terms "operation," "surgery," and "reoperation." We reviewed abstracts published in the English language for relevance, and studies addressing the primary question were selected for review and classification. A consensus on the rating of each paper was made as a group, and thus, interobserver reliability is not reported. The data were placed into an evidentiary table with recommendations made based on the volume and class of data. No attempt was made to judge the quality of the paper beyond the classification method described.

Based on previously published methodology,[1,15] the classification of articles and basis of review were as follows: Class I evidence, prospective randomized controlled trials; Class II, clinical series with prospective data collection or retrospective analysis of reliable data (observational studies, cohort studies, case control, prevalence studies); Class III, retrospectively collected data (clinical series, registries, databases); and Class IV evidence, anecdotal (case reports, testimony, theory, common sense). Recommendation were categorized by degree of certainty: standards: accepted patient management with a high degree of certainty (Class I or very strong Class II evidence); guidelines: strategy with moderate clinical certainty (Class II or preponderance of Class III evidence); and options: suggestions that may guide future research (Class III evidence)

\section{RESULTS}

The Medline search yielded 11,914 articles written from 1966 through March 1998 in which the key words "glioblastoma" and "astrocytoma" were used. Cross referencing with the key words "operation," "surgery," and "reoperation" limited the result to 1270 articles. This subset then underwent abstract review. From these references, 14 articles were found to contain data addressing reoperation of malignant glioma, and these were classified and the results tabulated in an evidentiary table (Table 1). We found no prospective, randomized controlled trials (Class I) directly addressing the issue of reoperation. Three studies were found in which the data were collected prospectively, either as a portion of a randomized trial addressing another issue or as part of an ongoing treatment strategy compared to historical controls (Class II). The 11 remaining studies were case series that included at least 10 patients and from which reoperation information could be extracted (Class III). Nonrelevant articles, case reports and opinion papers were omitted from further consideration for our purposes, although undoubtedly these contained interesting and potentially useful clinical information. 
Authas QYear Data

Authors \& Year

Description of Study

Class

Concl usions

Pool, 1967

Young, et al, 1981

Saloman, et al, 1982

Am mirati, et al, 1987

Harsh, et al, 1987

Moser, 1988

Vick, et al, 1989

Walner, et al, 1989

Berger, et al, 1991

retro review of 70 pts who under ment 2 nd craniot for Grades III \& IV tumors

Dirks, et al, 1993

retro review of 43 pts who under went 2 nd craniot after craniot \& XRT for Grade IV tumors

Strömblad, et al, 1993

Rostomily et al, 1994

Durmaz, et al, 1997 retro review of 46 pts; 16 under ment 2nd craniot for Grade IV tum ors

Sipos \& Afra, 1997 craniot for tumors of all grades

retro review of $24 \mathrm{pts}$ who under went 2 nd craniot for Grades III \& IV tumors

pro analysis of $40 \mathrm{pts}$ who underment 2 nd op as part of larger cohort

retro review of 55 pts who under went 2 nd craniot for Gades III \& IV tumors

retro review of $70 \mathrm{pts}$ who under went 2 nd craniot for Grades III \& IV tumors

retro review of select series of 11 pts who under went 2nd craniot onl y after sym ptomatic recurrence

retro review of $15 \mathrm{pts} w / K P S>60$ who under ment 2nd craniot for Grades III \& IV tumors

retro review of 39 pts who under went 2 nd IV tumors retro review of $52 \mathrm{pts}$ who under went 2 nd

III craniot w/attention to CT for Gades III \&

III

III

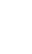

median sur wi wal for AA mas 88 wks \& 36 whs for GBM; time before deterioration correlated to age \& initial KPS score but not suru wal

III authors condude that reop in selected sym ptom atic, mell-defined lesions can im prove $Q O L$

III KPS score undhanged byop; median survival of 10 mks for GBM, 24 mks for AA after 2nd op; com pared w/historical controls, authors condude that reop doutles survival in selected pts

survival correlated wigrade; trend was seen $w /$ higher preop KP $S$, to tal resect $\&$ frontal lobe location; CT had no predictive value

III

time to deterioration in KP S score after 2nd op correlated to: time between ops, score $>70$ at 2 nd op, extent of re sect, \& younger age; survival only correlated to time to tumor progression

III median sur wi wal of 19 mks after 2 nd op; length of survival correlated to time between 1st \& 2nd op; com pared whistorical controls, supports role of reop; authors condude reop provides modest but significant increase in survival

II no significant change in sur wival when controlled for age \& KP S soore in group undergoing reop; suggests selection tias is a significant factor

II reop prolonged time to progression but not survival time; suggests im provement in $\mathrm{QQ}$; posto $p$ tumor wolume did not affect response to chemo

III unatle to demonstrate increase in mean survival w/multivariate analysis; appears to suggest selection tias is sgnificant factor

III survival dependent on tumor grade at 1st op \& interval to recurrence; authors generally fawor reop in selected pts

\footnotetext{
"AA $=$ anaplastic astrocytoma; chemo = chemotherapy; craniot = craniotom $y ; C T=$ computerized tomography; pro =

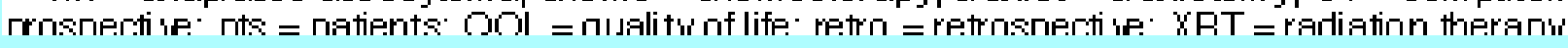


Based on this review the following conclusions could be made: 1) there is insufficient evidentiary data in the literature for the creation of a treatment standard; 2) there is insufficient evidentiary data in the literature for the creation of a guideline; and 3) reoperation in patients with recurrent malignant glioma is an option that may benefit select patients. Whether a second operation at the time of recurrence in patients with malignant glioma prolongs survival is unclear. However, there is evidence to suggest that reoperation may improve quality of life. Nonetheless, the role of reoperation in malignant glioma is not well delineated and should be considered for incorporation into the planning of clinical trials that address adjuvant therapy either in case-controlled or randomized fashion.

\section{DISCUSSION}

The value of reoperation in the management of recurrent malignant glioma remains unclear. Review of the available literature does demonstrate that reoperation for high-grade astrocytoma and glioblastoma can be accomplished with relatively low surgical mortality and morbidity rates. If we limit our inclusion criteria to only prospective data, we would conclude that reoperation can prolong time to progression,[9] which implies better quality of life, but the effect on overall patient survival time is not clear.[9,10,12] Unfortunately, even the prospective studies are questionable on the issue of reoperation alone, because none was specifically designed to address this issue in isolation. Adding the Class III studies would allow us to also suggest that patients either maintain their current performance or improve following a second procedure. A Karnofsky Performance Scale (KPS) score of at least 60 to 70 at the time of reoperation appears to affect the time to progression.[3,16] Overall, the authors of one Class II[10] and 10 Class III studies[2-4,6-8,11-14,16] suggest that operation for recurrent tumor benefits the patient, either through prolonged survival when compared with historical controls or in delaying the time to progression. However, results from two Class II[9,12] and one Class III[5] articles were unable to demonstrate a survival advantage when compared with similar patients controlled for KPS scores and age or by using multivariate analysis. All of the studies are biased by uncontrolled patient selection, leaving us to conclude that although there is evidence to support the role of reoperation in selected patients, this evidence at best can provide a treatment option. Guidelines and standards for appropriate patient selection must await additional study.

Evidenced-based review of the medical literature is beneficial in reviewing the available papers on a given topic of interest, as well as in pointing out the significant difficulty of comparing various reports and various groups within the same study. It also provides a methodology for ranking and assigning a weight to individual reports. Our search was limited to articles published with at least English-language abstracts. It is possible that valuable studies published in the international literature were omitted inadvertantly. It is also conceivable that additional articles of relevance did not come to our attention becuase of the limitations of our search criteria. We intended to address a relatively straightforward issue and were quickly surprised to find the overwhelming influence of selection bias apparent in the reported series. There was also a surprising lack of prospectively collected data; the majority of studies available represented retrospective case series. Whereas it is impractical to address many clinical issues with randomized trials, the treatment of malignant glioma is particularly suited to randomization because the overall treatment response is so poor, particularly in Grade IV recurrent tumors. It would appear likely that, with more sophisticated treatment protocols under development, the issue of reoperation alone as a factor could be addressed, at least as a case-controlled issue. 
There is insufficient evidence to formulate either a treatment standard or guideline on the role of reoperation in malignant glioma. Reoperation can be accomplished with acceptable morbidity rates and appears to benefit selected patients. The prospective data available conflict with respect to overall survival, but time to progression (quality of life) appears to be enhanced with reoperation in selected patients. These issues need to be addressed in the context of prospective clinical trials using case-controlled or randomized protocols.

\section{References}

\section{American Medical Association: Attributes to Guide the Development of Practice}

Parameters/Guidelines. Chicago: American Medical Association/Specialty Society, Practice Parameters Partnership, 1996

2. Ammirati M, Galicich JH, Arbit E, et al: Reoperation in the treatment of recurrent intracranial malignant gliomas. Neurosurgery 21:607-614, 1987

3. Berger MS, Tucker A, Spence A, et al: Reoperation for glioma. Clin Neurosurg 39:172-186, 1992

4. Dirks P, Bernstein M, Muller PJ, et al: The value of reoperation for recurrent glioblastoma. Can J Surg 36:271-275, 1993

5. Durmaz R, Erken S, Arslantas A, et al: Management of glioblastoma multiforme: with special reference to recurrence. Clin Neurol Neurosurg 99:117-123, 1997

6. Harsh GR IV, Levin VA, Gutin PH, et al: Reoperation for recurrent glioblastoma and anaplastic astrocytoma. Neurosurgery 21:615-621, 1987

7. Moser RP: Surgery for glioma relapse. Factors that influence a favorable outcome. Cancer 62:381-390, 1988

8. Pool JL: The management of recurrent gliomas. Clin Neurosurg 15:265-287, 1968

9. Rostomily RC, Spence AM, Duong D, et al: Multimodality management of recurrent adult malignant gliomas: results of a phase II multiagent chemotherapy study and analysis of cytoreductive surgery.

Neurosurgery 35:378-388, 1994

10. Salcman M, Kaplan RS, Ducker TB, et al: Effect of age and reoperation on survival in the combined modality treatment of malignant astrocytoma. Neurosurgery 10:454-463, 1982

11. Sipos L, Afra D: Re-operations of supratentorial anaplastic astrocytomas. Acta Neurochir 139:99-104, 1997

12. Strömblad LG, Anderson H, Malmström P, et al: Reoperation for malignant astrocytomas: personal experience and a review of the literature. Br J Neurosurg 7:623-633, 1993

13. Vick NA, Ciric IS, Eller TW, et al: Reoperation for malignant astrocytoma. Neurology 39:430-432, 1989

14. Wallner KE, Galicich JH, Malkin MG, et al: Inability of computed tomography appearance of recurrent malignant astrocytoma to predict survival following reoperation. J Clin Oncol 7:1492-1496, 1989 
15. Woolf SH: Practice guidelines, a new reality in medicine. II. Methods of developing guidelines. Arch Int Med 152:946-952, 1992

16. Young B, Oldfield EH, Markebery WR, et al: Reoperation for glioblastoma. J Neurosurg 55:917-921, 1981

Manuscript received April 14, 1998.

Accepted in final form June 1, 1998.

Address reprint requests to: Timothy Ryken, M.D., Division of Neurosurgery, Department of Surgery, University of Iowa Hospitals and Clinics, 200 Hawkins Drive, Iowa City, Iowa 52242. email: tryken@aol.com. 